\title{
As contribuições de John Clauser para o primeiro teste experimental do teorema de Bell: uma análise das técnicas e da cultura material \\ (John Clauser's contributions to the early experimental tests of Bell's theorem: an analysis of techniques and material culture)
}

\author{
Wilson Fábio de Oliveira Bispo ${ }^{\varpi}$, Denis Francis Gilbert David ${ }^{2}$, Olival Freire Jr. ${ }^{2}$ \\ ${ }^{1}$ Instituto Federal de Educação, Ciência e Tecnologia Baiano, Salvador, BA, Brasil \\ ${ }^{2}$ Instituto de Física, Universidade Federal da Bahia, Salvador, BA, Brasil \\ Recebido em 15/3/2012; Aceito em 13/5/2013; Publicado em 19/9/2013
}

\begin{abstract}
Discutiremos as principais contribuições de John Clauser para a realização do primeiro experimento que testou o teorema de Bell, através de uma análise focada na cultura material, isto é, instrumentos e técnicas utilizadas. Além de uma breve biografia de Clauser, faremos também uma contextualização histórica buscando responder a seguinte pergunta: o primeiro experimento que testou o teorema de Bell poderia ter sido realizado anteriormente? A análise da cultura material e das técnicas nos mostrará que existiam impedimentos a que um experimento como aquele pudesse ter sido realizado anteriormente.
\end{abstract}

Palavras-chave: Bell, Clauser, teoria quântica, variáveis escondidas, cultura material.

We discuss John Clauser's main contributions to the first experiment dedicated to tests of Bell's theorem through an analysis of its material culture, namely the instruments and techniques that were used. In addition to a biographical note of Clauser and a presentation of the context of that experiment, we asked the following question: experiments on Bell's theorem could have been carried out before? Our analysis shows that there were obstacles related to the material culture for such an anticipation.

Keywords: Bell, Clauser, quantum theory, hidden variables, material culture.

\section{Introdução}

A teoria quântica (TQ) tem sido submetida aos mais severos testes experimentais e dentre estes ocupam lugar de destaque os testes com o teorema de Bell utilizando pares de fótons emaranhados. Um indicador da importância destes experimentos foi a concessão do prestigiado prêmio Wolf de Física de 2010 a John Francis Clauser, Alain Aspect e Anton Zeilinger, "por suas fundamentais contribuições conceituais e experimentais para os fundamentos da física quântica, especificamente uma série crescentemente sofisticada de testes das desigualdades de Bell ou extensões usando estados quânticos emaranhados". [1] Clauser tem o mérito de ter feito (junto com Stuart Jay Freedman) o primeiro teste bem sucedido do teorema de Bell, cujos resultados foram publicados em 1972. Alguns dados, apontados a seguir, indicam as circunstâncias excepcionalmente difíceis nas quais Clauser conduziu aquele experimento. Desde 1988 testes com as desigualdades de Bell são chamados de testes de terceira geração por usarem como fonte para a produção de pares de fótons emaranha-

\footnotetext{
${ }^{1}$ E-mail: wilsonfabio@gmail.com.

dos a conversão paramétrica descendente, processo pelo qual fótons emitidos por laser interagem com cristais não lineares produzindo o par de fótons emaranhados. Trata-se de fonte especialmente poderosa, que revolucionou a técnica destes experimentos. Antes, os testes de segunda geração, como aqueles conduzidos por Alain Aspect e publicados entre 1981 e 1982 [2-4], bem como aquele conduzido por E. Fry em 1976 [5], usavam laser sintonizável para excitar amostras atômicas e obter o par de fótons por decaimento do elétron excitado. O experimento conduzido por Clauser e Freedman [6] usou um método mais rústico para obter os pares emaranhados, excitando uma amostra atômica com feixes de elétrons com energia conhecida. Tanto os experimentos de segunda e terceira geração coletam dados suficientes para boas estatísticas em questão de minutos ou mesmo segundos. Clauser e Freedman precisaram manter o experimento estabilizado por cerca de 200 horas para obter dados suficientes para a análise. Clauser não só contribuiu para a realização do primeiro experimento que testou o teorema de Bell mas também com a primeira proposta de um experimento realizável - através 
do artigo conhecido pelo acrônimo CHSH, o qual será comentado adiante - e com a realização de um segundo experimento que também testou o teorema de Bell [7], só que desta vez Clauser o realizou sozinho.

A abordagem historiográfica adotada neste trabalho privilegia a história dos instrumentos [8] e das técnicas como parte da cultura material. Embora cultura material seja conceito tradicional na história, oriundo da arqueologia, e história de instrumentos seja praticada há bastante tempo no âmbito da história da ciência, esta abordagem foi renovada e dinamizada com os trabalhos de Peter Galison, especialmente aqueles relacionados à instrumentação em física de partículas ao longo do século XX no livro Image and Logic: The Material Culture of Microphysics [9, 10]. Ao focar seu estudo na instrumentação, ao invés da tradicional perspectiva centrada nas grandes teorias e grandes experimentos em física de partículas, Galison pôde lançar luzes sobre as subcomunidades científicas em torno destes instrumentos e suas culturas, bem como sobre a relativa autonomia de cada uma dessas subcomunidades no âmbito da comunidade maior da Física. Esta abordagem pode ser útil na formação de uma cultura em ciências em nosso país, especialmente pela valorização que traz às componentes experimental e instrumental. Isto pode renovar uma visão de ciência, presente na nossa cultura, que valoriza unilateralmente a teoria em detrimento destas outras componentes. ${ }^{\boldsymbol{\square}}$ Além de seu interesse intrínseco, esta abordagem nos permite responder questões de interesse histórico. No caso em estudo, sabemos que durante muito tempo existiu um preconceito na comunidade de físicos contra pesquisas em fundamentos da teoria quântica, em especial a pesquisa relacionada às variáveis escondidas [11], sendo natural portanto a seguinte pergunta: o experimento realizado por Clauser poderia ter sido realizado anteriormente? Verificando quando os instrumentos e as técnicas utilizadas neste experimento foram desenvolvidos nós podemos contribuir para uma resposta àquela questão. Antecipando conclusões, a análise da cultura material e das técnicas nos mostrará que existiam impedimentos a que um experimento como aquele realizado por Clauser e Freedman fosse realizado anteriormente.

Neste artigo discutiremos as principais contribuições de Clauser para a realização do primeiro experimento que testou o teorema de Bell, apresentando uma descrição focada numa análise dos instrumentos e das técnicas (excitação, detecção e contagem de fótons) utilizados. Na primeira seção faremos uma breve contextualização histórica - o artigo EPR, a contribuição de David Bohm e o teorema de Bell - e discutiremos as contribuições de Clauser para o surgimento dos primeiros testes experimentais do teorema de Bell, bem como a realização do primeiro destes testes com fótons. $\mathrm{Na}$ segunda seção apresentaremos uma breve biografia de Clauser, a qual mostrará que Clauser pagou um preço na sua carreira profissional pela dedicação a um tema que estava nas margens da pesquisa em física à época. Na terceira seção faremos uma análise dos instrumentos utilizados no experimento realizado por Clauser, explicando o que eram esses aparatos, como funcionavam, quais seus papeis no referido experimento. Por fim, a quarta seção tratará da análise das técnicas utilizadas e será seguida das conclusões.

\section{Um pouco de história}

Albert Einstein, Boris Podolsky e Nathan Rosen propuseram, em 1935, um experimento de pensamento (Gedankenexperiment), conhecido hoje pelo acrônimo EPR [13], com o qual buscaram evidenciar que a teoria quântica seria incompleta. Neste experimento duas partículas ("A" e "B") interagem e em seguida são separadas por certa distância. Einstein propõe medir o momento de A e através de certos cálculos, derivados da interação das partículas, obter o valor do momento de B. Alternativamente, ele sugere medir a posição de A, e por processo análogo obter a posição de B. Como as medidas hipotéticas foram feitas em A, e uma medição em uma partícula não deveria alterar o estado de outra partícula distante, Einstein conclui que a partícula B tem posição e momento simultaneamente bem definidos. Como a teoria quântica interdita esta conclusão, pelo princípio da indeterminação de Heisenberg, Einstein conclui que a teoria quântica é uma teoria incompleta porque descreve menos propriedades que aquelas expressas pela partícula B. Bohr respondeu ao artigo de Einstein argumentando que o mesmo não havia levado em consideração as condições experimentais adequadas àquelas medições. $\mathrm{O}$ debate ficou inconcluso. Anos depois David Bohm introduziu certa simplicidade matemática no experimento EPR adequando-o a sistemas de dois níveis, a exemplo das componentes de spin, o que posteriormente proporcionou simplicidade na realização dos experimentos reais $[14,15] .^{\text {] }}$ De todo modo, o experimento EPR levou muito tempo sem despertar muita atenção da comunidade científica, situação que só se modificaria em meados da década de 1960 com o trabalho de Bell [16].

O teorema de Bell, por vezes considerado um dos resultados mais profundos do século XX [17, 18], pode ser expresso em uma família de resultados matemáticos que podemos resumir numa desigualdade. Bell chegou a esta desigualdade identificado certas premissas presentes na proposta EPR e traduzindo-as para uma linguagem matemática. As premissas, hoje conhecidas como realismo local, ou separabilidade, dizem que em um sistema composto por duas partes, estas têm propriedades

\footnotetext{
${ }^{2}$ A noção de cultura material foi utilizada por Cássio L. Vieira na reconstituição da história das emulsões nucleares, incluindo o trabalho de Cesar Lattes, ver Ref. [12]

${ }^{3}$ Devido a esta mudança alguns autores incluem o nome de Bohm no experimento de pensamento EPR, ver, por exemplo Ref. [3].
} 
bem definidas independente de medições e que medidas sobre uma das partes não devem alterar o estado da outra parte. Para construir suas desigualdades Bell expressou estas premissas introduzindo, na descrição do estado do sistema, variáveis adicionais, por isto ocultas, em relação àquelas adotadas pela teoria quântica. Disto surge a expressão teorias de variáveis ocultas locais (TVOL). Ao aplicarmos nesta desigualdade os valores obtidos pela Mecânica Quântica, a desigualdade é violada. De modo sucinto, o teorema de Bell diz que nenhuma TVOL pode reproduzir todos os resultados da teoria quântica. ${ }^{\mathbb{0}}$ Como veremos, Clauser esteve entre os primeiros, junto com Abner Shimony, a identificar que nenhum experimento já realizado permitia testar tal teorema, requerendo portanto novos experimentos para uma decisão entre as predições das TVOL e as da teoria quântica.

\section{Uma breve biografia de Clauser e suas contribuições}

O americano John Clauser nasceu em 1942, se graduou na Universidade de Berkeley e obteve o grau de Doutor na Universidade de Columbia com uma tese sobre medida experimental da radiação cósmica de fundo, sob orientação de Patrick Thaddeus. O treinamento no doutorado em medições de alta precisão da radiação eletromagnética certamente contribuiu para seus experimentos ulteriores, no campo da ótica, sobre o teorema de Bell [11, p. 589-90]. Durante seus estudos em Berkeley e Columbia, Clauser teve dificuldades em compreender a teoria quântica, dificuldades que ele argumenta que teria herdado do seu pai, Francis Clauser, um engenheiro aeronáutico que pesquisava física de fluidos e ainda segundo Clauser - não entendia esta teoria, apesar das similaridades entre a matemática dos fluidos e a da mecânica quântica. Clauser também argumenta que herdou de seu pai um saudável ceticismo, expresso no conselho para olhar sempre para os dados antes de tirar conclusões. Este conselho teria sido de grande valia, pois o resultado do primeiro experimento de Clauser não foi o que ele esperava com base na sua desconfiança em relação à teoria quântica. Clauser confiou nos dados encontrados e publicou seus resultados mesmo estes contradizendo suas expectativas iniciais.

As figuras chaves para a realização dos primeiros testes experimentais do teorema de Bell foram Clauser e Abner Shimony. Eles trabalhavam de modo independente sobre o tema e sem se conhecer. Quando Clauser publicou um resumo no Bulletin of the American Physical Society, sugerindo um experimento para testar o teorema de Bell, Shimony percebeu que alguém também trabalhava no tema e disse a Richard Holt e Michael Horne, estudantes de doutorado, "nós fomos ultrapas- sados" [21, p. 257]. Shimony então convidou Clauser para publicar um artigo juntamente com Holt e Horne, artigo hoje conhecido como $\mathrm{CHSH}$ devido as iniciais dos autores [22]. Nesse artigo eles desenvolveram o teorema de Bell, adequando-o a experimentos reais e apresentaram uma proposta de um experimento realizável que poderia testar este teorema. Após a publicação deste artigo, começaram a surgir os primeiros testes experimentais do teorema de Bell utilizando fótons óticos.

O primeiro teste experimental foi realizado por Clauser juntamente com Suart Jay Freedman (19442012) e teve seus resultados publicados em 1972. O tema da tese de doutorado de Freedman foi a realização deste experimento. Freedman faria uma carreira destacada como físico experimental, ocupando a Luis Alvarez Memorial Chair em física experimental na Universidade da Califórnia, Berkeley [23]. Uma das dificuldades enfrentada por eles - que não ocorreu nos testes experimentais posteriores - foi não conhecer qual deveria ser o resultado experimental que se buscava, pois, nas palavras do próprio Freedman "é uma grande ajuda quando se conhece qual deve ser o resultado experimental que se procura, quando não se sabe realmente se aquele é o resultado correto, pensa-se que seu experimento tem algum problema" [21, p. 285]. Vale ressaltar que Freedman e Clauser passaram dois anos testando, modificando e até construindo os aparatos para realizar este experimento que, após iniciado, demorou um período de dois meses para ser concluído [21, p. 267], além de realizá-lo "na idade da pedra do hardware", como declarou o próprio Clauser [21, p. 267]. O resultado experimental encontrado por Clauser e Freedman corroborou as previsões da teoria quântica e isto foi uma surpresa para Clauser, pois ele esperava "balançar o mundo" com a confirmação da existência das variáveis ocultas [11, p. 590].

Holt e Pipkin também realizaram um experimento, na Universidade de Harvard, na mesma época com a finalidade de testar o teorema de Bell, só que neste caso os resultados confirmavam as previsões das TVOL. Contudo eles não publicaram seus resultados, inseguros que estavam dos seus resultados, embora tenham veiculado os mesmos em um preprint [11, p. 593-599]. A conjectura de Clauser e Shimony [24] é que o problema estava no uso de um vidro opticamente ativo, o que teria influenciado o resultado experimental. Clauser então resolveu refazer o experimento realizado por Holt e Pipkin, só que desta vez ele o realizou sozinho, o que foi financeiramente cômodo, pois ele ainda tinha os aparatos utilizados no experimento anteriormente realizado com Freedman, só precisando trabalhar nas partes que eram diferentes como, por exemplo, a técnica de excitação. O resultado encontrado por Clauser foi contrário ao encontrado por Holt e Pipkin, criando as-

\footnotetext{
${ }^{4}$ Sobre as desigualdades de Bell ver: Pessoa Jr, O. Conceitos de Física Quântica, cap. XXVII [19]. Para um maior aprofundamento ver: Pessoa, cap. XXVIII, op. cit.[19]; Freire Jr. O.Ref. [20]; Bell Ref. [16] op. cit.

${ }^{5}$ Esta notícia biográfica de Clauser está apoiada na Ref. [11], salvo referência diversa.
} 
sim mais uma evidência contra as TVOL [11].

\section{Análise dos instrumentos do experi- mento de Clauser e Freedman ${ }^{\mathbf{W}}$}

Abaixo temos um esquema do experimento realizado por Clauser e Freedman representado na Fig. 1.

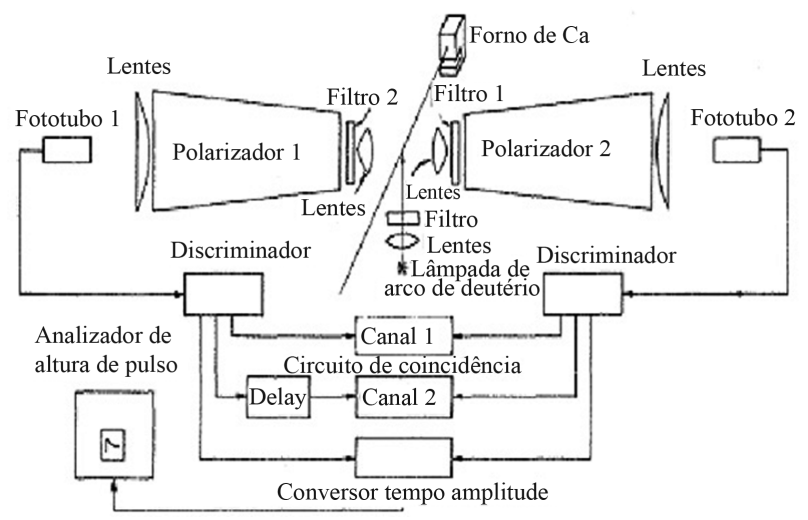

Figura 1 - Esquema do experimento realizado por Freedman e Clauser, in: Ref. [6, p. 939], (legendas traduzidas pelos autores desse trabalho). Um forno emitia um feixe de cálcio que era excitado por uma lâmpada de arco de deutério, emitindo assim pares de fótons emaranhados. Os fótons, após passar por lentes e polarizadores, eram detectados por fototubos, os quais transformavam os fótons detectados em pulsos elétricos. Esses pulsos após passar pelo circuito de coincidência, pelo analisador de altura de pulso e pelo conversor tempo amplitude, eram tratados e as medidas eram efetuadas.

O principal objetivo desse experimento foi verificar as previsões da teoria quântica e das TVOL e, a partir desta verificação, indicar qual das duas teorias estaria de acordo com os resultados experimentais encontrados. Nessa verificação mediu-se a correlação de polarização linear (grandeza que indica a direção de vibração do campo elétrico numa onda eletromagnética clássica) de dois fótons emitidos na desexcitação em cascata de átomos de cálcio ou mercúrio. Os dados obtidos foram aplicados nas equações encontradas no artigo que descreve o experimento e, por fim, o resultado encontrado foi comparado com as previsões das teorias. Comentaremos agora sobre os instrumentos usados no experimento.

Um forno de tântalo emitia um feixe de cálcio na forma de vapor, o qual era excitado por uma lâmpada de arco (também chamadas lâmpadas de descarga) de deutério através do fenômeno da absorção ressonante. Após a excitação, os fótons emitidos eram selecionados por filtros de interferência (ou interferômetros de Fabry-Pérot), que são dispositivos ópticos que refletem ou transmitem de maneira seletiva uma ou mais linhas espectrais estreitas da luz que incide sobre eles. Com a necessidade de concentrar o feixe no menor volume possível, para que seja aproveitado o maior número de átomos na saída do forno e aumentar assim a acumulação de dados, foram usadas lentes primárias anaesféricas - primárias por serem as primeiras a receber a luz vinda da fonte e anaesféricas por se afastarem do formato esférico para reduzir as aberrações esféricas (devido a esse efeito, onde deveria ser visto um ponto vê-se uma mancha).

Após a seleção dos fótons podendo formar um par correlacionado, realizava-se a seleção de suas polarizações (paralela ou perpendicular ao plano dos polarizadores), através de polarizadores do tipo "pilha de placas", que tinham dez lâminas de vidro inclinadas próximo ao ângulo de Brewster. Em cada lâmina ocorriam duas reflexões, uma em cada face, onde parte da luz que tinha certa polarização era refletida e a outra, com a polarização inversa, era transmitida. Um mecanismo que contém dois eixos paralelos interligados por uma cruz "Maltese" (ou cruz de malta) e por dois discos, chamado "Mecanismo Geneva", girava os polarizadores num incremento de $22,5^{\circ}$, para obter as condições mais favoráveis para testar as desigualdades de Bell, já que os conflitos mais nítidos entre a teoria quântica e as TVOL ocorriam para os ângulos de $22,5^{\circ}$ e $67,5^{\circ}$. Abaixo um esquema de um possível experimento para testar o teorema de Bell, representado na Fig. 2, apresentado por Clauser e Shimony [22, p. 1892].

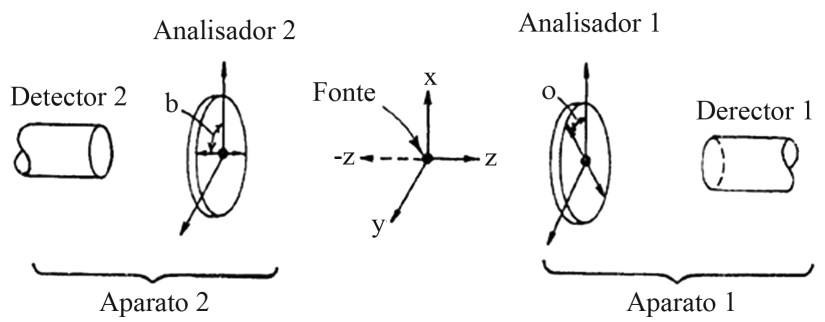

Figura 2 - Esquema de um experimento do teorema de Bell apresentado por Clauser e Shimony in Ref. [22, p. 1892], onde "a" e "b" representam os ângulos formados pelos eixos dos analisadores e os eixos de referência fixados.

A detecção era realizada através de tubos fotomultiplicadores, ou fototubos, do tipo "quanticons" (capazes de detectar fótons únicos), os quais absorviam a luz incidente sobre eles e emitiam elétrons (devido ao efeito fotelétrico). Estes elétrons se chocavam com dinodos e em cada um destes choques um elétron deslocava de 3 à 4 novos elétrons, que eram acelerados até o próximo dinodo, repetindo este processo sucessivamente, transformando assim a luz incidente em pulsos elétricos. No manual da Hamamatsu (empresa responsavel pela fabricação de fototubos) encontramos uma referência do primeiro tubo fotomultiplicador criado, o que ocorreu em 1930, sendo seus criadores Koller e Campbell [27].

Discriminadores, indicados logo após os fototubos na primeira figura, são circuitos que selecionam unicamente os sinais de interesse, aqui picos oriundos dos

\footnotetext{
${ }^{6}$ As seções 3 e 4 foram desenvolvidas na dissertação de mestrado de um dos autores (Bispo) [25] e uma versão preliminar foi apresentada na Ref. [26].
} 
tubos fotomultiplicadores, de maneira a eliminar eventos não válidos.

A contagem dos fótons era feita pelo circuito de coincidência - um aparato eletrônico que tinha por finalidade verificar se o par detectado era, de fato, o par correlacionado - através de uma janela de coincidência (tempo de duração entre as emissões dos dois fótons, que neste experimento foi de $8,1 \mathrm{~ns}$ ) existente entre os fótons detectados. O circuito de coincidência utilizado nesses experimentos foi desenvolvido inicialmente por Bruno Rossi (em 1930), trabalhando com física de raios cósmicos em Arcetri, Universidade de Florença. Sua fonte primária de inspiração foi o artigo de W. Bothe e W. Kolhörster, publicado na Z. Physik [28]. Rossi percebeu que o método de coincidência usado por Bothe e Kolhörster poderia ser bastante melhorado se fosse desenvolvido um método de gravar as coincidências, o que poderia proporcionar um melhor tempo de resolução, nascendo assim o circuito de coincidência clássico [29].

O Conversor Tempo-Amplitude media o tempo de atraso entre dois sinais emitidos pelos fototubos. Este tempo de atraso era utilizado para fazer o espectro de resposta (contagem realizada pelo circuito de coincidência em função do tempo entre dois eventos), o que verificava a validade dos eventos e assim o bom funcionamento do circuito de coincidência. O Analisador de Altura de Pulso era um dispositivo eletrônico que verificava a validade dos eventos, evitando que dois ou mais eventos simultâneos, mas casuais, sejam considerados coincidência. Normalmente um único fóton gera no fototubo uma corrente unitária "i", no caso de ocorrer dois eventos simultâneos não desejados, esta corrente dobra e assim esse dispositivo elimina esses eventos.

Finalmente, um segundo canal de contagem de coincidência foi utilizado para medir as coincidências acidentais. Este procedimento era realizado através de um circuito de retardo de eventos chamado "Delay". Este circuito era ligado entre um dos dois discriminadores e o circuito de coincidência, causando um atraso nas coincidências. Como estas coincidências tinham um retardo, não poderiam ser contadas como coincidências verdadeiras, logo estes dados seriam considerados coincidências acidentais, e deveriam ser subtraídos das coincidências totais.

\section{A análise das técnicas utilizadas no experimento}

Podemos dividir o experimento aqui analisado em três técnicas: de excitação, de detecção e de contagem de fótons. A primeira é caracterizada pela excitação dos átomos e emissão dos fótons correlacionados, representada pelos aparatos presentes em todo o processo de excitação - forno de tântalo, lâmpada de arco, etc. A segunda técnica é caracterizada pela seleção, detecção e transformação dos fótons em pulsos elétricos. Os instrumentos principais que compõem esta técnica são os fototubos, os filtros, as lentes e os polarizadores. A terceira técnica utilizada nos experimentos foi a da contagem de coincidência (ou técnica de coincidência), aqui representada pelo circuito de coincidência. Comentaremos agora sobre as particularidades de cada uma dessas técnicas.

$\mathrm{Na}$ técnica de excitação eles usaram uma desexcitação em cascata dos átomos de cálcio para a emissão do par de fótons correlacionados, a partir dos quais se media a correlação de polarização linear. Um feixe de cálcio, oriundo de um forno de tântalo, era excitado através do processo de absorção ressonante, no qual uma lâmpada de arco de deutério emitia um feixe contínuo de luz ultravioleta com comprimento de onda de $227,5 \mathrm{~nm}$. Antes de excitar o átomo de cálcio, a luz da lâmpada de deutério passava por lentes e filtros, para colimar e selecionar o feixe. A região de interação - local onde o cálcio interagia com a luz para realizar a excitação - consistia de um cilindro de vidro (Pyrex) de $5 \mathrm{~mm}$ de altura e $3 \mathrm{~mm}$ de diâmetro, onde a densidade de cálcio era $10^{10}$ átomos $/ \mathrm{cm}^{3}$. Os átomos de cálcio passavam do nível fundamental $4 s^{2}{ }^{1} S_{0}$ para o nível excitado $3 d 4 p^{1} P_{1}$. Na Fig. 3 vemos o esquema de decaimento em cascata apresentado por Freedman and Clauser.

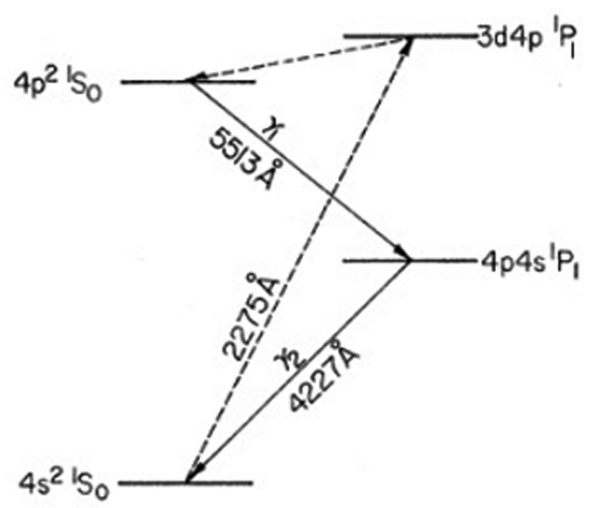

Figura 3 - Esquema do decaimento em cascata para o átomo de cálcio. A linha pontilhada mostra a trajetória de excitação partindo do estado inicial. Logo após temos dois decaimentos representados pela linha contínua, onde são emitidos os dois fótons $\gamma_{1}$ e $\gamma_{2}$ correlacionados em polarização com comprimentos de onda de $5513 \AA$ e $4227 \AA$. In: Ref. [6, p. 940].

Dos átomos que não decaíam diretamente para o estado fundamental, apenas $7 \%$ decaía para o estado $4 s^{2}{ }^{1} S_{0}$, a partir do qual ocorriam mais dois decaimentos (passando pelo estado intermediário $4 p 4 s^{1} P_{1}$ ) que davam origem ao par de fótons co-relacionados em polarização - esses fótons foram emitidos de uma cascata $J=0, J=1, J=0$ (onde $J$ é o momento angular total). A quantidade de dados coletados foi relativamente baixa comparada ao total de fótons emitidos. Como as conclusões do experimento dependem do tamanho da amostra estatística, a quantidade de dados foi fator fundamental para se obter um resultado experimental confiável. A duração do experimento foi de $200 \mathrm{~h}$. 
A técnica de detecção não se caracteriza apenas pela detecção em si, mas também pelas condições ideais para que ela ocorra. Essas condições estão relacionadas à seleção dos fótons com o devido comprimento de onda e devida polarização, mantendo o ambiente com baixa luminosidade e baixa intensidade do campo magnético. Os fótons emitidos passavam por lentes que tinham como objetivo reduzir o fenômeno da aberração esférica (no lugar de um ponto ver-se uma mancha), fenômeno este que poderia reduzir a eficiência da contagem. Como as emissões ocorriam em diversas direções, também foram usadas lentes colimadoras com certo ângulo de aceitação, aumentando a eficiência do experimento e reduzindo a possibilidade de apenas um dos detectores contar um único fóton pertencente ao par, perdendo assim a medida. Outra necessidade foi a de se selecionar os fótons que realmente pertenciam ao par correlacionado. A primeira seleção era feita com os filtros de interferência, onde só passavam os fótons com os devidos comprimentos de onda. A segunda pelos polarizadores, onde se escolhia a polarização correta dos fótons. Se a polarização fosse perpendicular com a outra, indicando pares de fótons correlacionados, a contagem era efetuada, caso contrário não. Como as condições mais favoráveis para testar as desigualdades de Bell ocorrem nos ângulos $\varphi$ (ângulo entre as orientações definidas nos polarizadores e os planos de polarização, ver Fig. 2) iguais a $22,5^{\circ}$ e $67,5^{\circ}$, os polarizadores giravam num incremento de $22,5^{\circ}$, para suprir esta necessidade. A detecção era concluída com a chegada dos fótons nos fototubos. Esses fototubos foram resfriados com o intuito de reduzir as taxas escuras, ou ruídos de fundo (são emissões espontâneas de elétrons do cátodo que ocorrem devido à temperatura dos fototubos).

$\mathrm{Na}$ técnica de contagem, cada fototubo estava ligado a um discriminador (que selecionava unicamente os sinais de interesse, eliminando sinais inválidos) e estes, por sua vez, estavam ligados a dois contadores de coincidência e a um conversor tempo-amplitude. O primeiro contador fazia a contagem de todas as coincidências. O segundo contador tinha um atraso em um dos canais de $50 \mathrm{~ns}$, para contar coincidências acidentais, as quais posteriormente seriam subtraídas das coincidências totais, obtendo-se assim a taxa de coincidências reais. Um conversor tempo-amplitude estava ligado a um analisador de altura de pulso, os quais mediam o tempo de atraso no perfil dos sinais elétricos desencadeados pela detecção de dois fótons. O resultado indicava o tempo de vida do estado intermediário ( $\sim 5 \mathrm{~ns})$. A taxa de coincidência dependia do feixe e da intensidade da lâmpada, o qual foi decrescendo gradualmente durante o experimento. O tempo de resolução de todo sistema foi de 1,5 ns. O curto tempo de vida do estado intermediário permitiu uma estreita janela de coincidência de 8,1 ns. Contagens de coincidências com janelas tão estreitas só se tornaram possível depois do desenvolvi- mento do transistor, em 1947 [30]. Anteriormente, a eletrônica baseada no uso de válvulas não era muito eficiente, pois trabalhava na frequência do mega hertz, tempo de resposta da ordem do micro segundo (apesar do magnetron ter sido desenvolvido antes da década de 1940 [31] e ser uma válvula que trabalha com alta freqüência, ele não poderia ter sido utilizado num circuito de coincidência por que não amplificava o sinal).

\section{Considerações finais}

John Clauser deve ser considerado um pioneiro no campo de fundamentos da teoria quântica por ter desempenhado um papel fundamental, tanto no terreno experimental quanto teórico, no estabelecimento do campo de pesquisas sobre o teorema de Bell, ou seja, sobre o emaranhamento quântico. Parece, portanto, justo que ele tenha partilhado o Prêmio Wolf de 2010 com Alain Aspect e Anton Zeilinger, os quais lideraram ulteriormente este campo.

No que pese a relevância das contribuições de Clauser para o campo de fundamentos da teoria quântica, ele mesmo não colheu no devido tempo os frutos do reconhecimento de sua atividade. Ao contrário, ele enfrentou obstáculos na sua carreira acadêmica que decorreram dos preconceitos existentes à época em relação ao tema dos fundamentos da teoria quântica e em especial contra o tema das variáveis ocultas. Estes obstáculos se expressaram, por exemplo, na não obtenção de uma posição permanente nas universidades norte-americanas. Por tais razões, em particular por ter enfrentado tais obstáculos, Clauser pode ser considerado um exemplo do que temos denominado de "dissidentes quânticos" [11, 32].

Quanto à cultura material, a instrumentação que permitiu a realização do primeiro experimento entre 1970 e 1972, podemos concluir que o fototubo e o circuito de coincidência já estavam disponíveis nesta época, pois tinham sido criados em 1930. A melhoria da eletrônica de modo a aprimorar o tempo de resolução no circuito de coincidência, contudo, só apareceria com a invenção do transistor em 1947. Ademais, mesmo com a invenção do transistor, a qualidade dos fototubos disponíveis não permitia a detecção de fótons únicos. Conforme depoimento de Freedman, os fototubos com esta sensibilidade tinham acabado de ser desenvolvidos quando ele e Clauser começaram a montagem do experimento: "a RCA tinha acabado de desenvolver estes tubos chamados de 'quanticons' e eu me tornei familiar com eles, de fato aprendi muito sobre fototubos" [21, p. 266]. O experimento foi realizado, portanto, aproximadamente na época que a técnica permitia sua realização, embora a motivação imediata para a sua realização não tenha sido este desenvolvimento da técnica mas sim o surgimento de um resultado teórico, o teorema de Bell, em meados da década de 1960. 


\section{Referências}

[1] http://wWw.wolffund.org.il/cat.asp?id=25\&cat_ Ectle=PHYSICS. Acessado em 4/3/2012.

[2] A. Aspect, J. Dalibard and G. Roger, Physical Review Letters 49, 1804 (1982).

[3] A. Aspect, P. Grangier and G. Roger, Physical Review Letters 49, 91 (1982).

[4] A. Aspect, P. Grangier and G. Roger, Physical Review Letters 47, 460 (1981).

[5] E.S. Fry and R.C. Thompson, Physical Review Letters 37, 465 (1976).

[6] S.J. Freedman and J.F. Clauser, Physical Review Letters 28, 938 (1972).

[7] J.F. Clauser, Physical Review Letters 36, 1221 (1976).

[8] D. Pestre, Cadernos IG/UNICAMP 6, 21 (1996).

[9] P. Galison, Image and Logic: The Material Culture of Microphysics (The University of Chicago Press, Chicago, 1997).

[10] P. Galison, in: A Ciência Tal Qual Se Faz, editado por F. Gil (Edições João Sá da Costa, Lisboa, 1999), p. 395-414.

[11] O. Freire Jr, Studies in History and Philosophy of Modern Physics 37, 577, (2006).

[12] C.L. Vieira, Um Mundo Inteiramente Novo se Revelou: Uma História da Técnica das Emulsões Nucleares (Livraria da Física, São Paulo, 2012).

[13] A. Einstein, B. Podolsky and N. Rosen, Physical Review 47, 777 (1935). Tradução de Cláudio Weber Abramo, Caderno de História e Filosofia da Ciência 2, 90 (1981).

[14] D. Bohm, Quantum Theory (Prentice Hall, New York, 1951).

[15] O. Freire Jr, David Bohm e a Controvérsia dos Quanta (Centro de Lógica, Epistemologia e História da Ciência da UNICAMP, Campinas, 1999).

[16] Bell, J.S. Physics 1, 195 (1964).

[17] J.F. Clauser, Quantum [Un]speakables: From Bell to Quantum Information", ed. by R.A. Bertlmann and A. Zeillinger (Springer, Berlin, 2002), p. 61.
[18] Aspect, A. Nature 398, 189 (1999).

[19] O. Pessoa Jr., Conceitos de Física Quântica (Livraria da Física, São Paulo, 2006), v. II, $1^{a}$ ed.

[20] O. Freire Jr., Caderno Catarinense de Ensino de Física 8, 212 (1991).

[21] L. Gilder, The Age of Entanglement: When the Quantum Physics was Reborn (Alfred A. Knopf, New York, 2008).

[22] J.F. Clauser, M.A. Horne, A. Shimony and R.A. Holt, Phys. Rev. Lett. 24, 549 (1970).

[23] R.N. Cahn and R.G. Hamish Robertson, Physics Today 66, 72 (2013).

[24] J.F. Clauser and A. Shimony, Rep. Prog. Phys. 41, 1881 (1978).

[25] W.F.O. Bispo, Sobre a Cultura Material dos Primeiros Testes Experimentais do Teorema de Bell: Uma Análise dos Instrumentos (1972-1982). Dissertação de mestrado, Universidade Federal da Bahia, 2009.

[26] W.F.O. Bispo e D.F.G. David, Teoria Quântica: Estudos Históricos e Implicações Culturais, editado por O. Freire Jr., O. Pessoa Jr. e J.L. Bromberg (EDUEPB, Campina Grande, 2010), pp. 95-105. Disponível no Scielo Livros: http://books.scielo.org/id/xwhf5.

[27] Autor? Photomultiplier Tubes, Basics and Applications, (Hamamatsu Photonics, Hamamatsu City, 1999) second edition.

[28] W. Bothe e W. Kolhörster, Z. Physik 56, 751 (1929).

[29] B. Rossi, Physics Today 34, 35 (1981).

[30] http://pt.wikipedia.org/wiki/ Trans\{\%\}C3\{\%\}ADstor Wikipédia, acessado em $7 / 3 / 2012$.

[31] http://www.fazano.pro.br/port62.htm], Acessado em $7 / 3 / 2012$.

[32] O. Freire Jr, Studies in History and Philosophy of Modern Physics 40, 280 (2009). Tradução para o português em O. Freire Jr., O. Pessoa Jr. e J.L. Bromberg (orgs) Teoria Quântica: Estudos Históricos e Implicações Culturais (EDUEPB, Campina Grande, 2010), pp. 33-64. Disponível no SciELO Livros: http: //books.scielo.org/id/xwhf5. 